\title{
Modes of Vesicle Retrieval at Ribbon Synapses, Calyx-Type Synapses, and Small Central Synapses
}

\author{
Ling-Gang Wu, ${ }^{1}$ Timothy A. Ryan, ${ }^{2}$ and Leon Lagnado ${ }^{3}$ \\ ${ }^{1}$ National Institute of Neurological Disorders and Stroke, Bethesda, Maryland 20892, 2 Department of Biochemistry, Weill Cornell Medical College, New \\ York, New York 10021, and ${ }^{3}$ Medical Research Council Laboratory of Molecular Biology, Cambridge CB1 0QH, United Kingdom
}

Key words: endocytosis; ribbon synapses; calyx of Held; hippocampal synapses; fusion; kiss-and-run

\section{Introduction}

Endocytosis is essential for the maintenance of synaptic transmission because it recycles and thus prevents complete depletion of synaptic vesicles in the nerve terminal. The goal of understanding synaptic vesicle recycling mechanisms was launched in earnest in 1973 when John Heuser and Tom Reese published their landmark electron microscope images of synaptic terminals of the frog neuromuscular junction (NMJ) (Heuser and Reese, 1973) back-to-back with those of the group of Bruno Ceccarelli (Ceccarelli et al., 1973). Soon after these publications, however, a debate arose regarding this issue, which was summed up by John Heuser in a later review article (Heuser, 1989a): "Two schools of thought have developed, one holding that vesicles acquire tracers directly via a reversible exo/endocytotic sequence in which they consistently maintain their biochemical identity during their transient continuity with the plasma membrane, the other holding that synaptic vesicles acquire tracers indirectly, via the formation of clathrin-coated vesicles which are spatially and temporally separate from exocytosis and reverse a temporary loss of the vesicles' individual identity upon merger with the plasma membrane." In total, at least three forms of endocytosis have been hypothesized to operate at synapses (Fig. 1). As indicated above, in the best characterized form, a full collapse of vesicle occurs with the plasma membrane during exocytosis, followed by clathrin-mediated invagination and fission during endocytosis (Heuser and Reese, 1973; Heuser, 1989b). This form is called full collapse fusion and retrieval. "Kiss-and-run" exocytosis and endocytosis involves fusion followed rapidly by fission without full collapse of vesicle membrane (Ceccarelli et al., 1973; Fesce et al., 1994). The original tracer experiments of Heuser and Reese also revealed that, after heavy stimulation, an endosome-like intracellular structure transiently appears, from which vesicles bud off (Heuser and Reese, 1973). Endosome-like structures are likely the result of bulk endocytosis, retrieval of a large piece of membrane directly from the plasma membrane (Koenig and Ikeda,

Received July 31, 2007; revised Aug. 13, 2007; accepted Aug. 24, 2007.

This work was supported by the National Institute of Neurological Disorders and Stroke Intramural Research Program (L.-G.W.) and grants from the National Institute of Neurological Disorders and Stroke and National Institute on Drug Abuse (T.A.R.) and the Medical Research Council (L.L.).

Correspondence should be addressed to Ling-Gang Wu, National Institute of Neurological Disorders and Stroke, Bethesda, MD 20892. E-mail: wul@ninds.nih.gov.

D01:10.1523/JNEUROSCI.3471-07.2007

Copyright $\odot 2007$ Society for Neuroscience 0270-6474/07/2711793-10\$15.00/0
1989, 1996; Takei et al., 1996; Teng and Wilkinson, 2000; Richards et al., 2000; Holt et al., 2003; Wu and Wu, 2007).

Among these three forms of endocytosis, kiss-and-run fusion and retrieval might provide two advantages over other forms of fusion and retrieval. First, in principle it could allow for rapid and economical vesicle recycling. Second, its narrow fusion pore could limit the rate of transmitter discharge out of the vesicle, resulting in a slower and smaller quantal response (Klyachko and Jackson, 2002; He et al., 2006). Switching between kiss-and-run and full collapse fusion has been proposed as a mechanism to regulate synaptic strength (Choi et al., 2000). Because of these potentially important physiological roles, kiss-and-run has been intensively studied with imaging and electrophysiological techniques in the past decade. However, at most synapses studied, it remains controversial as to whether kiss-and-run is a major form of endocytosis or whether it even exists. This is primarily because rapid fusion pore opening and closure are experimentally difficult to record directly. Consequently, the relative contribution of three forms of endocytosis in various physiological conditions remains unsettled. Here we review the modes of endocytosis, their underlying mechanisms, and relative contributions in three different types of synapses, including ribbon synapses of sensory neurons, calyx-type synapses, and small central synapses. The majority of materials discussed here will be presented as a symposium entitled The Debate on the Kiss-and-Run Mode of Fusion at Synapses at the 37th Annual Meeting for the Society of Neuroscience.

The electrophysiological and imaging techniques often are capable of monitoring the key feature of endocytosis, the time course of the membrane, or the vesicle protein uptake. One of the hypothetical differences between full collapse and kiss-and-run mode of fusion and retrieval is the rate of endocytosis. It is traditionally thought that kiss-and-run is rapid, whereas full collapse retrieval is slow. Consequently, rapid and slow endocytosis were interpreted as kiss-and-run and full collapse fusion, respectively. Recent studies raise the possibility that rapid endocytosis might also follow full collapse fusion (see the next section). Evidence in addition to the endocytosis time course is therefore needed to distinguish between kiss-and-run or full collapse fusion and retrieval. Because a large number of previous studies deal with the rate of endocytosis, we will discuss how the rate of endocytosis is regulated in a large part of our review. We will then discuss how we interpret these data in respect to the modes of endocytosis. 

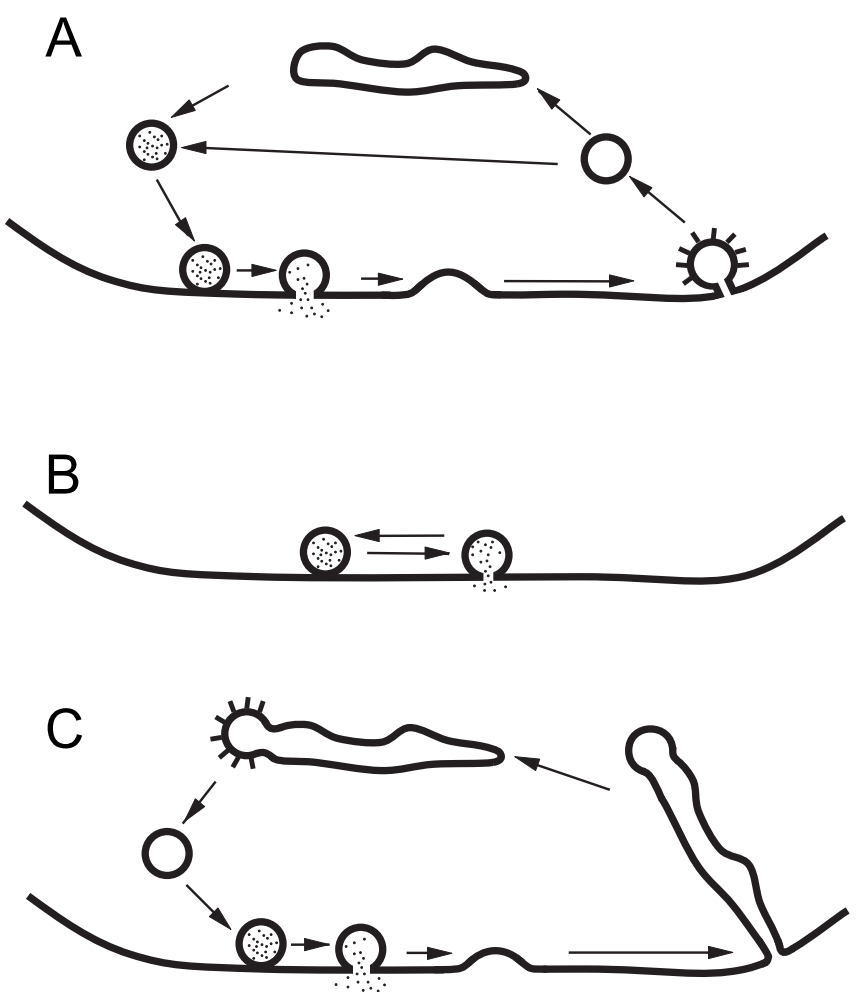

Figure 1. Three forms of endocytosis. $A$, Full collapse fusion and retrieval. $B$, Kiss-and-run fusion and retrieval. C, Bulk endocytosis.

\section{Ribbon synapses of sensory neurons}

Uncertainties about the properties of endocytosis at the synapse are attributable, at least in part, to the difficulties associated with measuring this process. One of the most direct approaches is the capacitance technique, which allows changes in membrane surface area to be recorded in real time, but this method cannot be applied to most synapses because of their small size (Penner and Neher, 1989). The solution to this problem is to use a neuron with a very large synaptic terminal, and a very useful example is found in the retina of goldfish, the depolarizing bipolar cell (von Gersdorff and Matthews, 1994a). This neuron can be isolated and attached to a glass coverslip, which also allows the synaptic vesicle cycle to be studied using a variety of imaging techniques, including wide-field fluorescence microscopy (Lagnado et al., 1996), total internal reflection fluorescence microscopy (TIRFM) (Zenisek et al., 2000), and interference reflection microscopy (IRM) (Llobet et al., 2003).

In most neurons, exocytosis is triggered by an action potential lasting a few milliseconds or less, but retinal photoreceptors and bipolar cells have "ribbon" synapses with unique features: as well as releasing neurotransmitter with extraordinary voltage sensitivity and speed, they support a continuous cycle of exocytosis and endocytosis that is modulated by "graded" changes in membrane potential (Lagnado et al., 1996; Rieke and Schwartz, 1996; Rea et al., 2004). High rates of continuous exocytosis are associated with a unique structure at the active zone, the "ribbon," to which vesicles attach by short filaments (Lenzi and von Gersdorff, 2001). Ribbon synapses also occur in hair cells of the ear and therefore transmit everything that we can see and hear.

The large size of bipolar cell terminals is related to their physiology: a large reservoir of vesicles allows high rates of exocytosis to be supported over prolonged periods (Lagnado et al., 1996). The replenishment of this reservoir requires that synaptic vesicles are efficiently retrieved from the cell surface, and at least three mechanisms can be distinguished: fast and slow endocytosis into small vesicles and bulk retrieval of larger chunks of membrane (summarized in Table 1).

Fast and slow modes of endocytosis

von Gersdorff and Matthews (1994a) were the first to directly demonstrate the speed of vesicle retrieval at the synapse. After a brief stimulus that released vesicles docked at the active zone, all the excess membrane was retrieved with a time constant of $\sim 1 \mathrm{~s}$. Neves and Lagnado (1999) then found that longer stimulation also causing release of vesicles from the reserve pool was followed by membrane retrieval occurring in two phases: fast endocytosis was followed by retrieval with a time constant of $10 \mathrm{~s}$ or more (Fig. 2). As more vesicles are released, increasing the proportion of the excess membrane is retrieved by slow endocytosis and the remainder by fast, but the rate constant of these processes does not alter. This last observation is notable because it indicates that endocytosis is modulated by changes in the route of retrieval rather than changes in the rate of a single process. The idea that fast and slow endocytosis in bipolar cell terminals are mechanistically distinct is also supported by the observation that slow retrieval is selectively inhibited by raising the hydrostatic pressure inside the terminal (Heidelberger et al., 2002), whereas fast retrieval is inhibited by raising the chloride concentration (Hull and von Gersdorff, 2004).

\section{Modulation by calcium}

Fast and slow modes of endocytosis can also be distinguished by their dependence on $\mathrm{Ca}^{2+}$. Limiting the spread of $\mathrm{Ca}^{2+}$ from the active zone by introducing calcium chelators causes a proportion of vesicles released by a brief stimulus to be retrieved by slow endocytosis rather than fast (Neves et al., 2001). Variations in the amplitude of the $\mathrm{Ca}^{2+}$ current also have the effect of altering the proportion of membrane retrieved by fast and slow mechanisms, again without modulating the intrinsic rate of these processes. It therefore seems that the $\mathrm{Ca}^{2+}$ microdomain triggering fast exocytosis is one of the signals that select vesicles for the fast route of retrieval. Vesicles that are not recycled by this mechanism are retrieved by the slower one, which operates at resting levels of $\mathrm{Ca}^{2+}$.

The capacitance response to stimuli lasting seconds often exhibits a pronounced delay between the closure of calcium channels and the rapid fall in capacitance, leading to the suggestion that fast endocytosis might be inhibited while global $\left[\mathrm{Ca}^{2+}\right]$ is high (von Gersdorff and Matthews, 1994b). A proper evaluation of this idea is difficult, because exocytosis continues for some time after a prolonged stimulus and the capacitance technique only measures the net change in membrane surface area (Neves et al., 2001). Nonetheless, when exocytosis (measured with FM1-43 [N-(3-triethylammoniumpropyl)-4-(4-(dibutylamino)styryl) pyridinium dibromide]) is compared with the net change in membrane surface area (measured by using the capacitance technique), it does appear that the fast mode of endocytosis is inhibited during a long stimulus (Neves and Lagnado, 1999).

Fast and slow modes of endocytosis have also been observed by applying the capacitance technique to the ribbon synapse of inner hair cells from the cochlea of the mouse (Moser and Beutner, 2000; Beutner et al., 2001). Fast endocytosis is also triggered by releasing caged $\mathrm{Ca}^{2+}$ to achieve free concentrations above 15 $\mu \mathrm{M}$ but surprisingly is not apparent after a depolarizing stimulus. A key question for the future is to establish whether fast endocytosis operates at ribbon synapses when they are activated by phys- 
Table 1. Comparison of endocytic mechanisms in the synaptic terminal of retinal bipolar cells

\begin{tabular}{lll}
\hline & Fast & Slow \\
\hline Predominates after stimulus that is... & Weak & Strong \\
Time constant & $1-2 \mathrm{~s}$ & $10-15 \mathrm{~s}$ \\
Clathrin dependent? & No & Yes \\
Actin dependent? & No & No \\
Calcium dependent? & Yes & No \\
Compartment & Small vesicle & Small vesicle \\
Selective? & Yes: does not compensate when slow endocytosis is & No: retrieves vesicles when fast endocytosis is \\
& blocked & blocked \\
Blocked by & Ca ${ }^{2+}$ buffers raised $\left[\mathrm{Cl}^{-}\right]$ & Increased hydrostatic pressure
\end{tabular}

PI-3, Phosphatidylinositol-3.
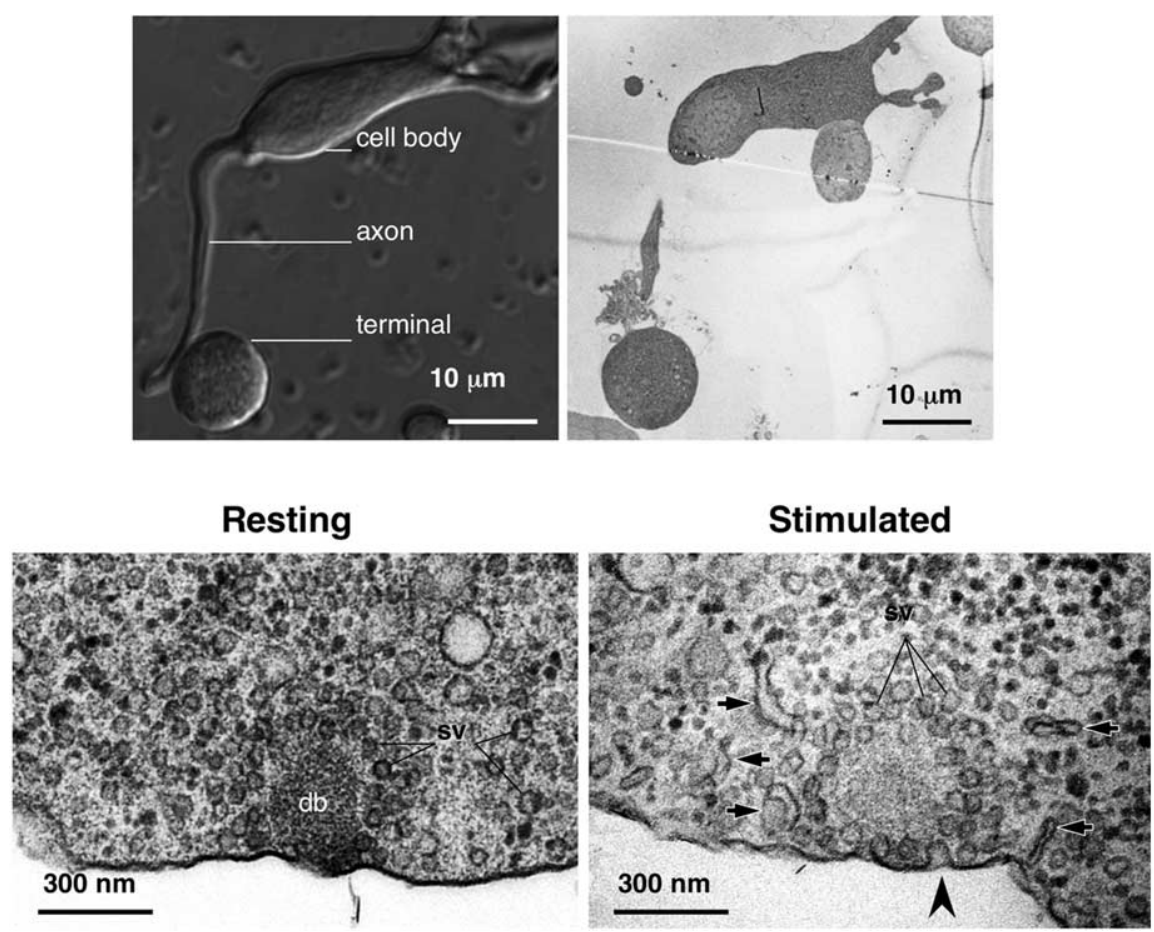

Figure 2. The giant synaptic terminal of retinal bipolar cells. Top left shows a dissociated bipolar cell, and on the right is an electron micrograph of the same cell. Below is the active zone in a resting and stimulated terminal. Note the electron-dense ribbon (also known as the dense body). Vesicles attach to the ribbon and also pack the surrounding cytoplasm. Note that, after 1 min of stimulation, bulk retrieval occurs at the margins of the active zone. The large arrow marks an omega shape, probably reflecting retrieval of a small vesicle at the active zone.

iological stimuli. A sensory stimulus normally causes only partial activation of voltage-sensitive calcium channels in these neurons, and it is not clear whether this is sufficient to trigger fast retrieval because experiments on isolated cells have tended to use stimuli that activate the calcium current maximally (Burrone and Lagnado, 2000).

\section{Slow endocytosis is clathrin dependent}

The idea that different kinetic components of membrane retrieval in bipolar cells represent distinct endocytic mechanisms has been confirmed by recent studies aimed at identifying their molecular basis (Jockusch et al., 2005). These studies have begun by testing the role of clathrin-mediated endocytosis (CME). CME retrieves membrane and surface proteins in a wide variety of cell types by the formation of small invaginations stabilized by a "cage" formed by a lattice of clathrin triskelia (Pearse et al., 2000; Royle and Lagnado, 2003). The formation of these coated invaginations also involves a number of accessory proteins, and these, together with clathrin, are enriched at synaptic terminals, includ- ing ribbon synapses of bipolar cells (Sherry and Heidelberger, 2005). It has long been known that CME operates at synapses, such as the NMJ (Heuser and Reese, 1973), but its speed and importance relative to other mechanisms have been difficult to establish. To investigate these questions, we introduced peptides and protein domains designed to interfere with defined interactions of clathrin and a key accessory protein, amphiphysin. Three of these interventions selectively inhibited the slow mode of endocytosis (Fig. 3) (Jockusch et al., 2005): the N-terminal "foot" of clathrin that binds accessory proteins, the domain of amphiphysin 1 that binds clathrin, and the domain of amphiphysin 1 that binds the AP2 adaptor (which recruits clathrin to the membrane). Thus, slow retrieval with a time constant of 10-15 s occurs by CME. The corollary of these observations is that fast retrieval with a time constant of $1 \mathrm{~s}$ occurs by a mechanism that is independent of clathrin.

Fast and slow modes of endocytosis in bipolar cells are not completely distinct, because both depend on the activity of a GTPase. It seems very likely that this is dynamin, which uses the energy from GTP hydrolysis to constrict and break membranes and was first characterized in the shibire mutant of Drosophila that is defective in synaptic vesicle recycling (Koenig et al., 1989). Dynamin is involved in the scission of membrane compartments during both clathrindependent and clathrin-independent mechanisms of endocytosis (Nichols and Lippincott-Schwartz, 2001).

Fast endocytosis is not kiss-and-run

The concept of fast recycling at the synaptic terminal is often taken to be synonymous with "kiss-and-run," a model of vesicle fusion that is distinguished by the idea that vesicles connect to the plasma membrane to release their contents without collapsing into the surface (Fesce et al., 1994). One form of the kiss-and-run model proposes that this connection is a proteinaceous pore that opens to release the neurotransmitter and then rapidly closes before the vesicle is retrieved (Klingauf et al., 1998; Aravanis et al., 2003; Gandhi and Stevens, 2003). The practical advantages of the bipolar cell terminal have allowed the testing of this model using methods that are more direct than those applied to small syn- 


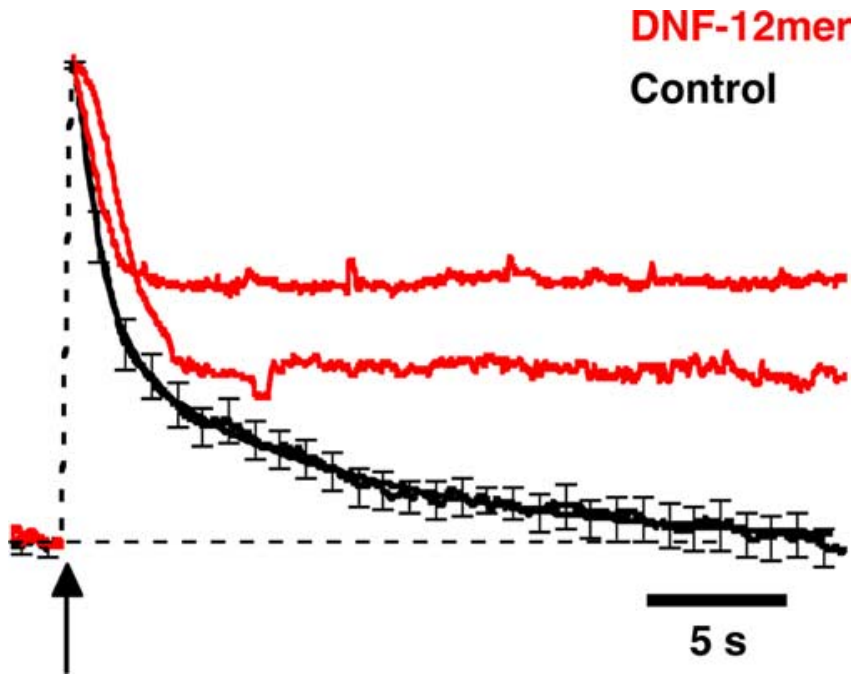

Figure 3. The slow phase of endocytosis is clathrin dependent. Capacitance responses to a depolarizing stimulus lasting $100 \mathrm{~ms}$. Normally, fast and slow phases of endocytosis are apparent, but infusion of a peptide designed to inhibit the interaction between amphiphysin and clathrin selectively blocks the slow phase (adapted from Jockusch et al., 2005). DNF-12mer refers to a 12 mer containing the DNF motif of amphiphysin 1, which binds the $\alpha$ subunit of AP2 with a high affinity.

apses. Zenisek et al. (2002) tested for kiss-and-run by using TIRFM to visualize directly the fusion of individual vesicles labeled with a membrane dye (FM1-43) and the subsequent diffusion of the dye in the surface of the synaptic terminal. Fusion was always followed by complete loss of FM1-43 from the vesicle, suggesting a free exchange of lipids between the vesicle and the plasma membrane. Llobet et al. (2003) tested for kiss-and-run in bipolar cells using a different approach; instead of looking for partial loss of vesicle contents, they investigated whether vesicles collapse by directly monitoring expansion of the surface membrane using IRM. Brief stimuli caused vesicles to merge into the surface, and this excess membrane was retracted with a time constant of $1 \mathrm{~s}$, reflecting fast endocytosis. The results of TIRFM and IRM therefore argue strongly against kiss-and-run as an important mechanism of exocytosis in retinal bipolar cells.

It therefore seems that fast endocytosis in the terminal of bipolar cells retrieves membrane that has collapsed into the surface but without formation of a clathrin coat. We do not yet know the molecular basis of fast endocytosis in ribbon synapses, except that it is likely to involve dynamin and one or more proteins that recruit dynamin to the membrane. A key question will be to identify the molecules that alter the shape of the membrane to make a vesicle. A number of proteins other than clathrin have now been shown to interact with membrane lipids to deform the bilayer, most notably proteins containing ENTH domains, such as epsin (Nossal and Zimmerberg, 2002), or BAR domains, such as endophilin (Zimmerberg and McLaughlin, 2004). Some of these bind preferentially to membranes with a certain degree of curvature, leading to the suggestion that they will be recruited sequentially, at the appropriate time and place to drive endocytosis forward (Lee and Schekman, 2004).

\section{Bulk retrieval and the actin cytoskeleton}

Ribbon synapses also support a mechanism of bulk retrieval into large membrane compartments that can be labeled by the uptake of large fluorescent markers that travel in the fluid phase (Lenzi et al., 2002; Paillart et al., 2003). Bulk retrieval is most obvious after stimulation lasting tens of seconds and is blocked by inhibitors of phosphatidylinositol-3 kinase, when endocytosis into small vesicles takes over all membrane recycling (Holt et al., 2003). In electron micrographs, these compartments appear as long invaginations of the surface membrane, often in the region around the active zone, and after internalization appear as C-shaped cisternae. The formation and movement of these cisternae is dependent on actin polymerization, whereas the retrieval of small vesicles is not.

In many synapses, vesicles interact with the actin cytoskeleton through the action of synapsins (Chi et al., 2001), and clathrin and actin colocalize at the endocytic regions that surround active zones (Roos and Kelly, 1999). However, ribbon synapses lack synapsins (Von Kriegstein et al., 1999), and capacitance measurements indicate that most basic aspects of the vesicle cycle in bipolar cells are unaffected by disruption of the actin cytoskeleton using cytochalasin D and latrunculin B, including fast exocytosis, fast endocytosis, and refilling of the rapidly releasable pool of vesicles (Holt et al., 2003). Thus, the actin cytoskeleton regulates bulk retrieval but not other aspects of vesicle recycling. The lack of an interaction between vesicles and the cytoskeleton may be a factor contributing to the unusually high mobility of vesicles in ribbon synapses of retinal bipolar cells (Holt et al., 2004) and cones (Rea et al., 2004).

\section{The calyx of Held synapse}

Capacitance measurement provides a quantitative estimate of vesicle fusion and retrieval

The calyx of Held is a glutamatergic nerve terminal containing $\sim 600$ conventional active zones (Sätzler et al., 2002). Its large size allows for whole-cell recordings of the capacitance from the whole nerve terminal (Sun and $\mathrm{Wu}, 2001$ ). Three pieces of evidence suggest that the whole-cell capacitance measurement at the calyx can be used to quantitatively study vesicle fusion and retrieval at this synapse. First, the capacitance jump is linearly proportional to the EPSC amplitude recorded at the same synapse when the postsynaptic glutamate receptors are not saturated (Sun and $\mathrm{Wu}, 2001$ ). Second, the capacitance jump induced by a single vesicle fusion is similar to the estimate of the membrane capacitance based on the size of the vesicle and the specific membrane capacitance of the biological membrane (Sun et al., 2002). Third, although the calyx is connected with an axon, both simulation and experiments show that the axon does not significantly affect the measurement of the capacitance jump at the calyx of Held (Sun et al., 2004).

The time course of endocytosis depends on stimulation intensity Recent studies at the calyx of Held suggest that the time constant of endocytosis varies as a function of the stimulus intensity. In general, after mild stimulation, such as an action potential-like stimulus, the time constant of endocytosis is brief, in the range of $<0.5$ to $\sim 10$ s (Sun et al., 2002; Wu et al., 2005; Yamashita et al., 2005). After an intermediate level of stimulation, such as a $10-20$ ms depolarization or a train of 10-20 action potential-like stimuli at a high frequency, the time course of endocytosis become slow, ranging from a few seconds to $\sim 20 \mathrm{~s}$ (Sun and Wu, 2001; Sun et al., 2002; Wu et al., 2005; Yamashita et al., 2005). After intense stimuli, such as 10 pulses of $20 \mathrm{~ms}$ depolarization at $1-10$ $\mathrm{Hz}$ or 200 action potential-like stimulus at a high frequency, in addition to a slow time course of endocytosis a rapid component of endocytosis with a time constant of $1-2 \mathrm{~s}$ is activated (Fig. $4 \mathrm{~A}$ ) (Wu et al., 2005). Except for the fast component of endocytosis after intense stimuli, the time constant of endocytosis is linearly proportional to the amount of exocytosis (Sun and Wu, 2001; 

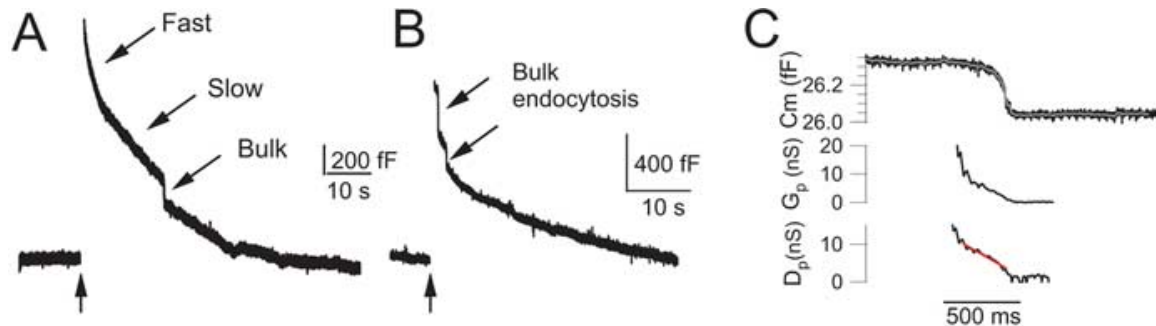

D

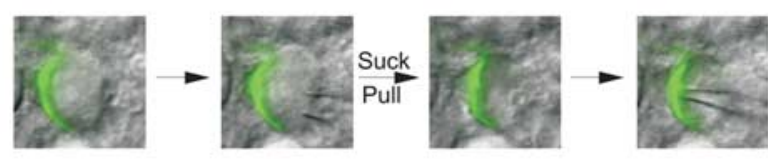

$E$
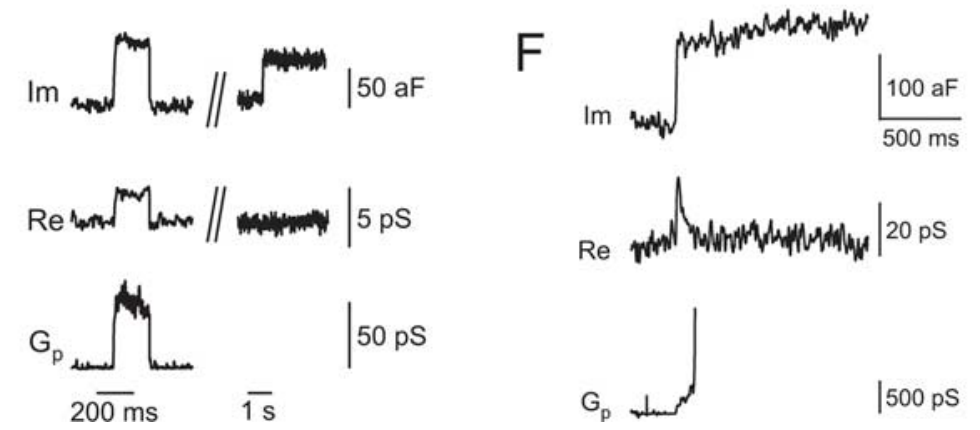

$50 \mathrm{pS}$

$\operatorname{Re}$
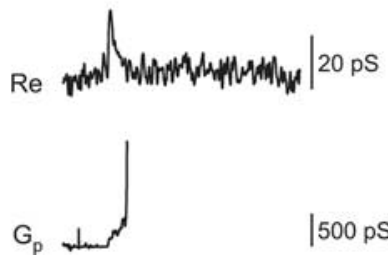

Figure 4. Endocytosis at the calyx of Held. $A$, Three kinetic forms of endocytosis (arrows), rapid, slow, and bulk endocytosis, were observed by whole-cell capacitance $\left(C_{m}\right)$ recordings at the calyx of Held. The stimulus (vertical arrow) was 10 depolarizing pulses of $20 \mathrm{~ms}$ from -80 to $+10 \mathrm{mV}$ at $10 \mathrm{~Hz}$. $\boldsymbol{B}$, The whole-cell membrane capacitance $\left(C_{\mathrm{m}}\right)$ response to 10 depolarizing pulses at $10 \mathrm{~Hz}$ (vertical arrow). The arrows indicate bulk endocytosis that occurred within a few seconds after the stimulus. $C$, The whole-cell membrane capacitance $\left(C_{m}\right)$, the fission pore conductance $\left(G_{\mathrm{p}}\right)$ and the fission pore diameter $\left(D_{\mathrm{p}}\right)$ during bulk endocytosis. The $20-80 \%$ decrease of $D_{\mathrm{p}}$ was fit with a linear regression line (red) with a slope (red) of $-31 \mathrm{~nm} / \mathrm{s}(C) . C_{\mathrm{m}}$ trace was low-pass filtered at $30 \mathrm{~Hz}$ (gray), from which $G_{\mathrm{p}}$ and $D_{\mathrm{p}}$ were calculated. $\boldsymbol{A}-\boldsymbol{C}$ are adapted from Wu and Wu (2007). D, The procedure to perform cell-attached recordings at the release face of a calyx. A calyx associated with a postsynaptic neuron was first identified (left). The calyx was loaded with Lucifer yellow (green). The postsynaptic neuron was sucked and pulled away by a pipette shown in the middle left (middle 2 panels). Another pipette was positioned at the release face of the calyx membrane (right) for cell-attached recordings, from which individual vesicle fusions, such as those shown in $\boldsymbol{E}$ were obtained. $\boldsymbol{E}$, Im (imaginary component of the admittance, reflecting capacitance), Re (real component of the admittance, reflecting conductance), and the fusion pore conductance $\left(G_{p}\right)$ during a capacitance flicker (left) and an up step that was not followed by a down step within $2 \mathrm{~s}$. The capacitance flicker with a detectable fusion pore conductance indicates kiss-and-run fusion. The nonflicker up step (right) occurring 10 s later was not accompanied by detectable Re changes, suggesting a full collapse fusion with the fusion pore that is too fast and/or too large to resolve. $F, I m, R e$, and $G_{p}$ during a full collapse fusion. Note that $G_{p}$ was detected in this fusion event. D-F are adapted from He et al. (2006).

Wu et al., 2005; Yamashita et al., 2005). This is consistent with the idea that the capacity of endocytosis is saturated after a mild stimulus (Sankaranarayanan and Ryan, 2000).

Activation of rapid endocytosis after intense stimuli could not be explained by saturation of the endocytosis capacity. Addition of a calcium buffer, EGTA, blocks this rapid form of endocytosis, suggesting that an increase of the intracellular calcium concentration triggers rapid endocytosis (Wu et al., 2005). This form of endocytosis may play a significant role during high-frequency stimulation (Wu et al., 2005). Its capacity may reach approximately eight vesicles per second at or near an active zone ( $\mathrm{Wu}$ et al., 2005). Its role after stimulation seems less significant as during stimulation, likely because of the fall of the intracellular calcium concentration after stimulation (Wu et al., 2005). The rapid component could reflect kiss-and-run fusion and retrieval, although other explanations are possible (see the section regarding ribbon synapses). The slow component is consistent with clathrin-mediated slow endocytosis, although experimental evidence is still needed to confirm this possibility at the calyx of Held
$1500 \mathrm{pS}$

synapse. Slow endocytosis is mediated by dynamin at the calyx of Held (Yamashita et al., 2005), consistent with results obtained at many other synapses (Koenig and Ikeda, 1989; Palfrey and Artalejo, 1998; Jockusch et al., 2005; Newton et al., 2006).

\section{Bulk endocytosis}

In addition to the smooth rapid and the slow component of the capacitance decay that reflect rapid and slow endocytosis, the capacitance could also drop rapidly by 20 $500 \mathrm{fF}$ within $\sim 30-500 \mathrm{~ms}$ (Fig. $4 A, B$ ) (Wu and $\mathrm{Wu}, 2007$ ). Such an abrupt drop of the capacitance reflects the instant of bulk membrane retrieval from the plasma membrane. Approximately $10 \%$ of exocytosed vesicles can be retrieved via this form of endocytosis during intense stimulation condition, consistent with the electron microscopic observation of large vacuoles at this same synapse (de Lange et al., 2003). The contribution of bulk endocytosis might be much larger because only very large membrane structures could be detected with the whole-cell capacitance recording technique ( $\mathrm{Wu}$ and $\mathrm{Wu}, 2007$ ). During bulk endocytosis, the decrease in the fission pore conductance could be detected, from which we estimated that the fission pore diameter decreased at $\sim 39$ $\mathrm{nm} / \mathrm{s}$ (Fig. 4C) (Wu and $\mathrm{Wu}, 2007)$. The rate of the fission pore closure is independent of the size of the bulk membrane structure ( $\mathrm{Wu}$ and $\mathrm{Wu}, 2007$ ), raising the possibility that the fission pore closure rate measured during bulk endocytosis could be extrapolated to small vesicles. The frequency of bulk endocytosis peaked $(\sim 0.021 \mathrm{~Hz})$ in $<10 \mathrm{~s}$ after stimulation and decayed with a half time $<20$ s, indicating that the time course of bulk endocytosis is much faster than previously estimated with low time-resolution techniques (Fig. 4B) (Wu and $\mathrm{Wu}, 2007)$. Our results also suggest that bulk endocytosis is composed of two kinetically different steps: the abrupt drop of the capacitance that reflects the fission process, and the time between stimulation and the fission process, during which membrane invagination leads to the fission pore formation. The fission pore formation seems to take a much longer time than the fission pore closure process. Thus, the time course of endocytosis seems to be primarily controlled by membrane invagination that forms the fission pore.

\section{Kiss-and-run and full collapse fusion and retrieval revealed by cell-attached recordings}

Rapid endocytosis, as shown in whole-cell recordings from the whole nerve terminal, is consistent with the kiss-and-run mode of fusion. However, the signature of kiss-and-run is a transient fusion pore opening and closing, as detected with the cellattached capacitance recording technique in endocrine cells (Lindau and Alvarez de Toledo, 2003). By pulling out the postsynaptic neuron using a large pipette (Fig. $4 D$ ), cell-attached 
capacitance recordings were made at the exposed release sites of the calyx of Held (He et al., 2006). Individual capacitance up steps were observed during high-potassium application. The size of up steps is similar to the capacitance of a single vesicle estimated with electron microscopy, suggesting that these up steps reflect single vesicle fusion. The up step is blocked by a protease trypsin, which blocks exocytosis but not the calcium current (He et al., 2006), suggesting that the up step is not caused by a calcium-dependent but exocytosis-independent artifact, as observed in the whole-cell configuration (Wu et al., 2005; Yamashita et al., 2005). A small fraction of up steps are followed within $2 \mathrm{~s}$ by a down step of the same size (Fig. 4E) (He et al., 2006). Such a pair of up and down steps is called a capacitance flicker. The capacitance flicker duration ranges from $10 \mathrm{~ms}$ to $2 \mathrm{~s}$, with a mean of $\sim 300 \mathrm{~ms}$ (He et al., 2006). For most capacitance flickers, the fusion pore conductance is too large to measure (He et al., 2006). It is estimated to be larger than 288 pS (He et al., 2006). In a small fraction of capacitance flickers, however, a fusion pore conductance ranging from 15 to $288 \mathrm{pS}$ with a mean of $\sim 66 \mathrm{pS}$ is observed (Fig. $4 E$ ), which may correspond to a fusion pore with a mean diameter of $\sim 1.1 \mathrm{~nm}$ (He et al., 2006). As shown by simulation, such a small fusion pore would generate a slow and small miniature EPSC compared with full collapse fusion (He et al., 2006). Indeed, a small fraction of miniature EPSCs were slow and small, consistent with a role of kiss-and-run in controlling the quantal response (He et al., 2006). These results suggest that a minor fraction of fusion events are kiss-and-run during high-potassium application. It is unclear to what extent kiss-and-run contributes to endocytosis during more physiological stimulation conditions, such as the action potential trains.

Most capacitance up steps reflect full collapse fusion (He et al., 2006). They are not followed in a brief time by an equal size down step (Fig. 4E). Their initial fusion pores are often too large to resolve (Fig. $4 E$ ). However, in a small fraction of up steps, an initial fusion pore conductance of $\sim 250 \mathrm{pS}$ was resolved, which was followed in $\sim 10-300 \mathrm{~ms}$ by a rapid pore expansion (Fig. $4 F$ ) (He et al., 2006). These results provide kinetic evidence indicating the existence of full collapse fusion at synapses.

In summary, whole-cell capacitance recordings at the calyx of Held reveal three kinetically different forms of endocytosis: rapid, slow, and bulk endocytosis. The rate of fission pore closure during bulk endocytosis has been resolved. Cell-attached capacitance recordings at the release site of the calyx indicate that a minor fraction of fusion events are kiss-and-run fusion, whereas the majority is full collapse fusion during high-potassium application. Although the whole-cell and the cell-attached capacitance recording techniques are considered powerful techniques in resolving the time course of endocytosis and kiss-and-run fusion, it is technically difficult to apply to most conventional nerve terminals that are much smaller than the calyx of Held. In addition, cell-attached recordings require the formation of a tight seal over a small patch area. It is unclear whether such a procedure influences the incidence of kiss-and-run or full collapse fusion.

\section{Small central synapses}

Although the debate regarding mechanisms of vesicle recycling was first framed regarding endocytosis at the NMJ, it was relaunched at other synapses and particularly at the synapses formed between dissociated hippocampal and cortical neurons grown in cell culture beginning approximately one decade ago.
First measurements of the kinetics of endocytosis at small CNS terminals

The debate of how vesicles recycle at hippocampal neurons arose with the advent of vital imaging techniques (Betz and Bewick, 1992) that allowed one to determine the kinetics of the vesicle cycle in living nerve terminals after membrane depolarization (Ryan et al., 1993). In the first such measurements, membrane depolarization was achieved using hyperkalemic solutions, leading to the exocytosis of a large fraction of the vesicle pool. The reinternalization of vesicle membrane, followed in a pulse-chase manner using FM1-43, revealed that, after exocytosis, the recovery of membrane required $\sim 1 \mathrm{~min}$, similar to what had been reported for kinetic studies at the frog NMJ using electron microscopic imaging one decade earlier (Miller and Heuser, 1984). This rather slow retrieval timescale was generally thought to be incompatible with the potential needs of actively firing synapses, particularly at small CNS nerve terminals in which the total number of vesicles is only on the order of $\sim 100$. As a result, the original speculation of about the possibility of a direct retrieval route (kiss-and-run) at the NMJ led to a search for evidence of such a mode of endocytosis in hippocampal synapses. However, it is important to point out that it was impossible to tell from the early kinetic studies whether or not the observed slow timescale was a reflection of inherent properties of a minimum time required for clathrin-mediated endocytosis or whether it represented some other step that became rate limiting as a result of the large stimuli used to perform these first measurements.

\section{Evidence that some retrieval events occur rapidly}

The first evidence that some recycling events may occur faster than those reported initially compared the efficiency of destaining of different amphipathic fluorescent dyes from vesicles undergoing exocytosis. This idea, which posited that a kiss-and-run event might not allow dyes with slower dissociation rates to escape during release of neurotransmitter, was originally tested for low-frequency stimulation conditions and showed, however, that even dyes that differed by a factor of 10 in dissociation rates $(0.6$ vs $6 \mathrm{~s}$ ) revealed very similar effective rates of destaining (Ryan et al., 1996). Subsequent analysis of similar experiments for highfrequency stimulation revealed small but measurable differences in overall destaining efficiency (Klingauf et al., 1998; Pyle et al., 2000) when using different dyes, and this difference was interpreted as arising from kiss-and-run recycling events. This type of analysis however relies on the assumption that dye that is secreted does not readily rebind to the synaptic plasma membrane and get reinternalized, a problem that potentially would be exacerbated by dyes with tighter association with the outer leaflet of the plasma membrane.

Additional high-sensitivity measurements at the level of single vesicles also indicated that, during exocytosis, synaptic vesicles do not necessarily release all of previously loaded fluorescent dye (Aravanis et al., 2003; Richards et al., 2005). Although these data both argue that certain vesicle retrieval events likely occur on a timescale that prevents efficient release of amphipathic dyes, it is unclear whether this results from dye rebinding during vesicle recycling, a higher than expected affinity of the dye for some synaptic vesicle lumen component that slows its destaining, or bona fide kiss-and-run-like recycling events. There is also the possibility that endocytic events that occur on a $\sim 1 \mathrm{~s}$ timescale might not represent a separate mode of endocytosis but rather one end of the spectrum of the timescales associated with clathrin-mediated retrieval. 
pHluorin-based measurements of endocytosis

A significant improvement in measurements of vesicle recycling came about with the introduction of synapto-pHluorins ( $\mathrm{spH}$ ), a synaptic vesicle lumen-targeted green fluorescent protein whose $\mathrm{pKa}$ for quenching by protons is ideally suited for detecting the $\mathrm{pH}$ change of the synaptic vesicle as it undergoes exocytosis and subsequent endocytosis and reacidification (Miesenbock et al., 1998). In addition, this approach provided the first opportunity to examine the fate of specific synaptic vesicle proteins as they undergo exocytosis and endocytosis (Sankaranarayanan and Ryan, 2000). This approach, using vesicle-associated membrane protein-2 (VAMP-2) as the carrier protein for pHluorin, provided the ability to perform numerous measurements at much higher time resolution than previously possible at the same set of synaptic terminals. These initial studies demonstrated that the time course of endocytosis became significantly slower with increasing accumulation of VAMP-2 on the synaptic surface (Sankaranarayanan and Ryan, 2000) and indicated that the slow time course observed in the original experiments using FM dye uptake were likely operating in a regimen in which the endocytic machinery was saturated. For stimuli in the range of 50 action potentials, at which the system does not appear to be saturated, the endocytosis time is on the order of $\sim 20 \mathrm{~s}$.

One of the factors limiting the signal-to-noise in the original $\mathrm{spH}$ experiments was the fact that a small fraction of the expressed protein resides on the axonal and synaptic surface even in resting cells. Because every copy of $\mathrm{spH}$ on the surface is $\sim 25$ times brighter than those residing in the acidic vesicle, even a small percentage of expressed protein residing on the surface will create a large fluctuating background signal. Ghandi and Stevens (2003) photobleached this surface pool to improve the signal-tonoise ratio of their measurements, enabling them to study the fluorescence transients evoked by single action potentials. These studies, which examined the fluorescence decay of the pHluorin signal following single action potentials, came to the conclusion that three distinct modes of endocytosis are manifest at small CNS nerve terminals. The first, representing effectively instantaneous decays in fluorescence (at the time resolution of these experiments) were ascribed to kiss-and-run and also implied that the reacidification timescale is $\sim 50 \mathrm{~ms}$ or shorter. The second mode reflected a slower signal decay on the 10-15 s timescale, whereas the third mode represented signals that failed to decay and were interpreted as molecules of $\mathrm{spH}$ that became "stranded" on the synaptic surface. Subsequently, two different studies showed that the pool of VAMP that resides on the surface exchanges with that in vesicles during recycling (FernandezAlfonso et al., 2006; Wienisch and Klingauf, 2006), which significantly complicates the interpretation of the studies in which a prebleached surface is used. Furthermore, direct estimates of reacidification times using rapid surface quenching for recently endocytosed synaptic vesicles indicates that it occurs with a time constant of $\sim 4$ s (Atluri and Ryan, 2006; Granseth et al., 2006). In addition, recent use of pHluorin targeted to synaptophysin, which has a much lower surface fraction than when using VAMP-2, allowed Granseth et al. (2006) to examine responses to single action potentials without the need to prebleach the surface. There data showed only a single mode of endocytosis with a $\sim 14$ s time constant.

The molecular basis of synaptic vesicle recycling at small CNS nerve terminals

The advent of improved optical methods combined with conventional molecular genetics as well as the use of small interfering
RNAs has allowed the role of a number of proteins to be examined at small CNS nerve terminals. In particular, Granseth et al. (2006) used pHluorin-tagged synaptophysin combined with small interfering RNA directed against the clathrin heavy chain gene to show that endocytosis is completely blocked after brief rounds of stimulation, or at least slowed very significantly, when clathrin expression is significantly reduced. Analysis of vesicle recycling at cortical synapses formed between neurons derived from dynamin 1 knock-out mice revealed that, during highfrequency firing above $10 \mathrm{~Hz}$, all endocytosis is blocked (Ferguson et al., 2007). Thus, in these nerve terminals, a large fraction, if not all, of vesicle endocytosis appears to depend critically on two of the molecular players associated with classical, coated-vesiclemediated endocytosis.

\section{Is there a functional role for kiss-and-run?}

The main original motivation for postulating the existence of the kiss-and-run pathway was that the early experiments indicated that the coated-vesicle-mediated retrieval was occurring on a timescale thought to be incompatible with the needs of actively firing synapses. Although the current estimates for the time for recovery of this pathway is on the order of $\sim 14 \mathrm{~s}$, the question arises whether or not this is sufficient to accommodate the known release rates of such small nerve terminals under various stimulation conditions, particularly those in which release of the entire recycling vesicle pool might occur in this time frame. For lowfrequency stimulation, there is little threat of depleting the recycling pool, and therefore the demand for kiss-and-run from a kinetic standpoint would not rear its head. For highfrequency stimulation, the entire vesicle pool can be made to release neurotransmitter (Fernandez-Alfonso and Ryan, 2004), and, therefore, if recycling were too slow, vesicle depletion would ensue. If kiss-and-run were operating under these conditions, it could in principle sustain synaptic transmission. However, postsynaptic electrophysiological analyses of the efficacy of synaptic transmission under these conditions indicate that synaptic responses become severely depressed (Pyott and Rosenmund, 2002), and thus kiss-and-run, if it exists, does not seem to spare these synapses from the consequences of slow vesicle recycling. From the point of view of kiss-andrun, the most compelling case to examine at CNS synapses will be examples in which high release probability is maintained at high frequency for individual terminals. Such a set of parameters would either require unusually large pools or faster vesicle recycling as might be afforded by a kiss-and-run pathway.

\section{How should kiss-and-run be defined?}

The strongest evidence to date for the existence of kiss-and-run at small CNS synapses derives primarily from the fact that dyes loaded in a previous round of recycling apparently fail to completely escape during exocytosis. Experiments that do not rely on dye destaining but instead follow the fate of specific synaptic vesicle proteins after exocytosis do not find any significant evidence for kiss-and-run. Instead, results from these approaches indicate that synaptic vesicle recycling appears to depend explicitly on the function of endocytic proteins known to operate in the classical coated-vesicle-mediated retrieval pathway of endocytosis. To date, no experiments that support the presence of kissand-run in this system have been examined under conditions in which candidate endocytic molecules have been perturbed or depleted.

One of the assumptions in experiments that have searched for kiss-and-run is that events with a faster timescale than that re- 
Table 2. Three modes of endocytosis at three different synapses

\begin{tabular}{|c|c|c|c|}
\hline Mode of endo & Ribbon-type synapse & Calyx-type synapse & Small CNS synapse \\
\hline \multicolumn{4}{|l|}{ Full collapse } \\
\hline Evidence & Slow endo $0^{a, b}$ & $\begin{array}{l}\text { Slow endo } 0^{c, d} ; \text { capacitance up step with } \\
\text { fusion pore expansion }{ }^{e}\end{array}$ & Slow endo ${ }^{f, g}$ \\
\hline Predominance & Strong stim & Most stim & Most stim \\
\hline Clathrin dependence & Yes $^{h}$ & $?$ & Yes $^{i}$ \\
\hline \multicolumn{4}{|l|}{ Kiss-and-run } \\
\hline Evidence & Rapid endo ${ }^{a, b, j}$ & $\begin{array}{l}\text { Rapid endo }{ }^{d, k} \text {; capacitance flicker with a } \\
\text { detectable fusion pore conductance }{ }^{e}\end{array}$ & $\begin{array}{l}\text { Partial FM dye loss with faster kinetics }{ }^{l, m, n} ; \text { partial dye } \\
\text { retention }{ }^{o} \text {; fast pHluorin transient }\end{array}$ \\
\hline Predominance in & Mild stim & Mild, strong stim & Most stim \\
\hline Clathrin dependence & $\mathrm{No}^{h}$ & $?$ & $?$ \\
\hline Caveat or conflict & Rapid endo could follow full collapse ${ }^{q}$ & & $\begin{array}{l}\text { Partial dye loss might not equal to kiss-and-run; fast } \\
\text { pHluorin transient attributable to lateral diffusion }{ }^{i, r} \text {; } \\
\text { only slow pHluorin transient is observed }{ }^{i}\end{array}$ \\
\hline \multicolumn{4}{|l|}{ Bulk endocytosis } \\
\hline Evidence & $\begin{array}{l}\text { Large vacuoles under the } \mathrm{EM}^{\text {s. }} \text {; large } \\
\text { punctuates of dye uptake }\end{array}$ & $\begin{array}{l}\text { Large vacuoles under the } \mathrm{EM}^{u} \text {; large } \\
\text { capacitance down step with fission pore }\end{array}$ & Deep in-foldings of the plasma membrane ${ }^{w}$ \\
\hline Predominance & Strong stim & Strong stim & $?$ \\
\hline Clathrin dependence & $?$ & $?$ & $?$ \\
\hline
\end{tabular}

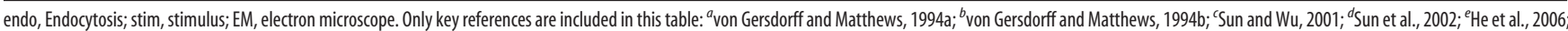

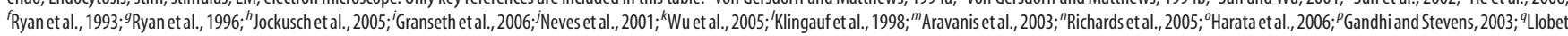

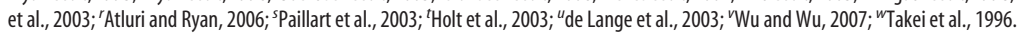

ported for the average coated-vesicle-mediated recovery route would necessarily imply the existence of a separate mode for endocytosis. However, these kinetic arguments assume that each event in the coated-vesicle-mediated recovery mechanism would occur with the same kinetics as the average. If this pathway were stochastic, then a distribution of timescales would be expected. Thus, it is possible that endocytic events that occur in $\sim 1$ s could be one extreme of a distribution of timescales in which the mean of all events is $14 \mathrm{~s}$. Because of these complications, identifying kiss-and-run events in this system will require showing that they represent a distinct class of retrieval that does not require the function molecules known to control the coated-vesicle pathway. It remains to be seen whether or not the evidence to date in favor of kiss-andrun in this system will satisfy these criteria.

\section{Bulk endocytosis at small nerve terminals}

In the first tracers experiments using HRP at the frog NMJ, Heuser and Reese documented the presence of transient internal organelles after stimulation, from which tracer-containing vesicles could be seen budding with clathrin coats. These data led to the speculation that essential events in remaking the synaptic vesicle might occur from an endosome instead of the plasma membrane. Ultrastructural analysis of cortical synaptosomes after intense stimulation showed the presence of complex internal organelles, but, in most cases, these appeared to be deep infoldings of the plasma membrane, and the lumen of the structures maintained continuity with the extracellular space (Takei et al., 1996). This view was supported by more recent electron microscopy coupled with tomography (Ferguson et al., 2007) that showed that, when endocytosis cannot keep up with exocytosis at small nerve terminals, such as in the dynamin 1 knock-out during high-frequency firing, a large number of coated pits connected to deep in-foldings that are contiguous with the plasma membrane are present. Thus, the prevailing evidence for small nerve terminals is that endocytosis of synaptic vesicles occurs directly at the plasma membrane and not in a bulk form from which later vesicle budding would take place.

\section{Conclusions}

The study of vesicle membrane and protein retrieval at nerve terminals has led to the hypothesis that several forms of endocytosis, including full collapse, kiss-and-run, and bulk endocytosis, might be operational. The evidence for these different modes at three different types of synapses is summarized in Table 2. It is generally agreed that full collapse endocytosis plays a significant role, and bulk endocytosis may become important after intense stimulation. Although a number of different methods have been used to characterize the kiss-and-run form of retrieval, the evidence is neither in complete agreement across different preparations or even within a preparation. Ultimately, it will be necessary to determine the molecular basis of vesicle retrieval for a variety of physiological stimuli at each of these types of synapses to determine how different forms of endocytosis have adapted different machineries to recycle vesicles and sustain neurotransmitter release. Only at that point can meaningful comparisons be made between recycling in a ribbon, that in a giant synapse and that in a small nerve terminal.

\section{References}

Aravanis AM, Pyle JL, Tsien RW (2003) Single synaptic vesicles fusing transiently and successively without loss of identity 423:643-647.

Atluri PP, Ryan TA (2006) The kinetics of synaptic vesicle reacidification at hippocampal nerve terminals. J Neurosci 26:2313-2320.

Betz WJ, Bewick GS (1992) Optical analysis of synaptic vesicle recycling at the frog neuromuscular junction. Science 255:200-203.

Beutner D, Voets T, Neher E, Moser T (2001) Calcium dependence of exocytosis and endocytosis at the cochlear inner hair cell afferent synapse. Neuron 29:681-690.

Burrone J, Lagnado L (2000) Synaptic depression and the kinetics of exocytosis in retinal bipolar cells. J Neurosci 20:568-578.

Ceccarelli B, Hurlbut WP, Mauro A (1973) Turnover of transmitter and synaptic vesicles at the frog neuromuscular junction. J Cell Biol 57:499-524.

Chi P, Greengard P, Ryan TA (2001) Synapsin dispersion and reclustering during synaptic activity. Nat Neurosci 4:1187-1193.

Choi S, Klingauf J, Tsien RW (2000) Postfusional regulation of cleft glutamate concentration during LTP at "silent synapses." Nat Neurosci 3:330-336.

de Lange RP, de Roos AD, Borst JG (2003) Two modes of vesicle recycling in the rat calyx of Held. J Neurosci 23:10164-10173. 
Ferguson SM, Brasnjo G, Hayashi M, Wolfel M, Collesi C, Giovedi S, Raimondi A, Gong LW, Ariel P, Paradise S, O’Toole E, Flavell R, Cremona O, Miesenbock G, Ryan TA, De Camilli P (2007) A selective activitydependent requirement for dynamin 1 in synaptic vesicle endocytosis. Science 316:570-574.

Fernandez-Alfonso T, Ryan TA (2004) The kinetics of synaptic vesicle pool depletion at CNS synaptic terminals. Neuron 41:943-953.

Fernandez-Alfonso T, Kwan R, Ryan TA (2006) Synaptic vesicles interchange their membrane proteins with a large surface reservoir during recycling. Neuron 51:179-186.

Fesce R, Grohovaz F, Valtorta F, Meldolesi J (1994) Neurotransmitter release: fusion or "kiss-and-run"? Trends Cell Biol 4:1-4.

Gandhi SP, Stevens CF (2003) Three modes of synaptic vesicular recycling revealed by single-vesicle imaging. Nature 423:607-613.

Granseth B, Odermatt B, Royle SJ, Lagnado L (2006) Clathrin-mediated endocytosis is the dominant mechanism of vesicle retrieval at hippocampal synapses. Neuron 51:773-786.

He L, Wu XS, Mohan R, Wu LG (2006) Two modes of fusion pore opening revealed by cell-attached recordings at a synapse. Nature 444:102-105.

Heidelberger R, Zhou ZY, Matthews G (2002) Multiple components of membrane retrieval in synaptic terminals revealed by changes in hydrostatic pressure. J Neurophysiol 88:2509-2517.

Heuser J (1989a) The role of coated vesicles in recycling of synaptic vesicle membrane. Cell Biol Int Rep 13:1063-1076.

Heuser JE (1989b) Review of electron microscopic evidence favouring vesicle exocytosis as the structural basis for quantal release during synaptic transmission. Q J Exp Physiol 74:1051-1069.

Heuser JE, Reese TS (1973) Evidence for recycling of synaptic vesicle membrane during transmitter release at the frog neuromuscular junction. J Cell Biol 57:315-344.

Holt M, Cooke A, Wu MM, Lagnado L (2003) Bulk membrane retrieval in the synaptic terminal of retinal bipolar cells. J Neurosci 23:1329-1339.

Holt M, Cooke A, Neef A, Lagnado L (2004) High mobility of vesicles supports continuous exocytosis at a ribbon synapse. Curr Biol 14:173-183.

Hull C, von Gersdorff H (2004) Fast endocytosis is inhibited by GABAmediated chloride influx at a presynaptic terminal. Neuron 44:469-482.

Jockusch WJ, Praefcke GJ, McMahon HT, Lagnado L (2005) Clathrindependent and clathrin-independent retrieval of synaptic vesicles in retinal bipolar cells. Neuron 46:869-878.

Klingauf J, Kavalali ET, Tsien RW (1998) Kinetics and regulation of fast endocytosis at hippocampal synapses. Nature 394:581-585.

Klyachko VA, Jackson MB (2002) Capacitance steps and fusion pores of small and large-dense-core vesicles in nerve terminals. Nature 418:89-92.

Koenig JH, Ikeda K (1989) Disappearance and reformation of synaptic vesicle membrane upon transmitter release observed under reversible blockage of membrane retrieval. J Neurosci 9:3844-3860.

Koenig JH, Ikeda K (1996) Synaptic vesicles have two distinct recycling pathways. J Cell Biol 135:797-808.

Lagnado L, Gomis A, Job C (1996) Continuous vesicle cycling in the synaptic terminal of retinal bipolar cells. Neuron 17:957-967.

Lee MC, Schekman R (2004) Cell biology. BAR domains go on a bender. Science 303:479-480.

Lenzi D, von Gersdorff H (2001) Structure suggests function: the case for synaptic ribbons as exocytotic nanomachines. BioEssays 23:831-840.

Lenzi D, Crum J, Ellisman MH, Roberts WM (2002) Depolarization redistributes synaptic membrane and creates a gradient of vesicles on the synaptic body at a ribbon synapse. Neuron 36:649-659.

Lindau M, Alvarez de Toledo G (2003) The fusion pore. Biochim Biophys Acta 164:167-173.

Llobet A, Beaumont V, Lagnado L (2003) Real-time measurement of exocytosis and endocytosis at synapses and neuroendocrine cells using interference reflection microscopy. Neuron 40:1075-1086.

Miesenbock G, De Angelis DA, Rothman JE (1998) Visualizing secretion and synaptic transmission with $\mathrm{pH}$-sensitive green fluorescent proteins. Nature 394:192-195.

Miller TM, Heuser JE (1984) Endocytosis of synaptic vesicle membrane at the frog neuromuscular junction. J Cell Biol 98:685-698.

Moser T, Beutner D (2000) Kinetics of exocytosis and endocytosis at the cochlear inner hair cell afferent synapse of the mouse. Proc Natl Acad Sci USA 97:883-888.

Neves G, Lagnado L (1999) The kinetics of exocytosis and endocytosis in the synaptic terminal of goldfish retinal bipolar cells. J Physiol (Lond) 515:181-202.

Neves G, Gomis A, Lagnado L (2001) Calcium influx selects the fast mode of endocytosis in the synaptic terminal of retinal bipolar cells. Proc Natl Acad Sci USA 98:15282-15287.

Newton AJ, Kirchhausen T, Murthy VN (2006) Inhibition of dynamin completely blocks compensatory synaptic vesicle endocytosis. Proc Natl Acad Sci USA 103:17955-17960.

Nichols BJ, Lippincott-Schwartz J (2001) Endocytosis without clathrin coats. Trends Cell Biol 11:406-412.

Nossal R, Zimmerberg J (2002) Endocytosis: curvature to the ENTH degree. Curr Biol 12:R770-R772.

Paillart C, Li J, Matthews G, Sterling P (2003) Endocytosis and vesicle recycling at a ribbon synapse. J Neurosci 23:4092-4099.

Palfrey HC, Artalejo CR (1998) Vesicle recycling revisited: rapid endocytosis may be the first step. Neuroscience 83:969-989.

Pearse BM, Smith CJ, Owen DJ (2000) Clathrin coat construction in endocytosis. Curr Opin Struct Biol 10:220-228.

Penner R, Neher E (1989) The patch-clamp technique in the study of secretion. Trends Neurosci 12:159-163.

Pyle JL, Kavalali ET, Piedras-Renteria ES, Tsien RW (2000) Rapid reuse of readily releasable pool vesicles at hippocampal synapses. Neuron 28:221-231.

Pyott SJ, Rosenmund C (2002) The effects of temperature on vesicular supply and release in autaptic cultures of rat and mouse hippocampal neurons. J Physiol (Lond) 539:523-535.

Rea R, Li J, Dharia A, Levitan ES, Sterling P, Kramer RH (2004) Streamlined synaptic vesicle cycle in cone photoreceptor terminals. Neuron 41:755-766.

Rieke F, Schwartz EA (1996) Asynchronous transmitter release: control of exocytosis and endocytosis at the salamander rod synapse. J Physiol (Lond) 493:1-8.

Richards DA, Guatimosim C, Betz WJ (2000) Two endocytic recycling routes selectively fill two vesicle pools in frog motor nerve terminals. Neuron 27:551-559.

Richards DA, Bai J, Chapman ER (2005) Two modes of exocytosis at hippocampal synapses revealed by rate of FM1-43 efflux from individual vesicles. J Cell Biol 168:929-939.

Roos J, Kelly RB (1999) The endocytic machinery in nerve terminals surrounds sites of exocytosis. Curr Biol 9:1411-1414.

Royle SJ, Lagnado L (2003) Endocytosis at the synaptic terminal. J Physiol (Lond) 553:345-355.

Ryan TA, Reuter H, Wendland B, Schweizer FE, Tsien RW, Smith SJ (1993) The kinetics of synaptic vesicle recycling measured at single presynaptic boutons. Neuron 11:713-724.

Ryan TA, Smith SJ, Reuter H (1996) The timing of synaptic vesicle endocytosis. Proc Natl Acad Sci USA 93:5567-5571.

Sankaranarayanan S, Ryan TA (2000) Real-time measurements of vesicleSNARE recycling in synapses of the central nervous system. Nat Cell Biol 2:197-204.

Sätzler K, Sohl L, Bollmann JH, Borst JGG, Frotscher M, Sakmann B, Lubke JH (2002) Three-dimensional reconstruction of a calyx of Held and its postsynaptic principal neuron in the medial nucleus of the trapezoid body. J Neurosci 22:10567-10579.

Sherry DM, Heidelberger R (2005) Distribution of proteins associated with synaptic vesicle endocytosis in the mouse and goldfish retina. J Comp Neurol 484:440-457.

Sun JY, Wu LG (2001) Fast kinetics of exocytosis revealed by simultaneous measurements of presynaptic capacitance and postsynaptic currents at a central synapse. Neuron 30:171-182.

Sun JY, Wu XS, Wu LG (2002) Single and multiple vesicle fusion induce different rates of endocytosis at a central synapse. Nature 417:555-559.

Sun JY, Wu XS, Wu W, Jin SX, Dondzillo A, Wu LG (2004) Capacitance measurements at the calyx of Held in the medial nucleus of the trapezoid body. J Neurosci Methods 134:121-131.

Takei K, Mundigl O, Daniell L, De Camilli P (1996) The synaptic vesicle cycle: a single vesicle budding step involving clathrin and dynamin. J Cell Biol 133:1237-1250. 
Teng H, Wilkinson RS (2000) Clathrin-mediated endocytosis near active zones in snake motor boutons. J Neurosci 20:7986-7993.

von Gersdorff H, Matthews G (1994a) Dynamics of synaptic vesicle fusion and membrane retrieval in synaptic terminals. Nature 367:735-739.

von Gersdorff H, Matthews G (1994b) Inhibition of endocytosis by elevated internal calcium in a synaptic terminal. Nature 370:652-655.

Von Kriegstein K, Schmitz F, Link E, Sudhof TC (1999) Distribution of synaptic vesicle proteins in the mammalian retina identifies obligatory and facultative components of ribbon synapses. Eur J Neurosci 11:1335-1348.

Wienisch M, Klingauf J (2006) Vesicular proteins exocytosed and subsequently retrieved by compensatory endocytosis are nonidentical 9:1019-1027.

Wu W, Wu LG (2007) Rapid bulk endocytosis and its kinetics of fission pore closure at a central synapse. Proc Natl Acad Sci USA 104:10234-10239.

Wu W, Xu J, Wu XS, Wu LG (2005) Activity-dependent acceleration of endocytosis at a central synapse. J Neurosci 25:11676-11683.

Yamashita T, Hige T, Takahashi T (2005) Vesicle endocytosis requires dynamin-dependent GTP hydrolysis at a fast CNS synapse. Science 307:124-127.

Zenisek D, Steyer JA, Almers W (2000) Transport, capture and exocytosis of single synaptic vesicles at active zones. Nature 406:849-854.

Zenisek D, Steyer JA, Feldman ME, Almers W (2002) A membrane marker leaves synaptic vesicles in milliseconds after exocytosis in retinal bipolar cells. Neuron 35:1085-1097.

Zimmerberg J, McLaughlin S (2004) Membrane curvature: how BAR domains bend bilayers. Curr Biol 14:R250-R252. 